\title{
Présentation d'un projet de pente d'eau à grand gabarit
}

\section{Description of a wide-gauge 'water-incline' elevator design}

\author{
B. Fauconnier et J.M. Manzone
}

Société générale de Technique et d'Études

La pente d'eau de par sa destination qui est de permettre aux bateaux de franchir une dénivellation, appartient à la famille des élévateurs à bateaux. Cependant, sa morphologie l'éloigne des deux types d'élévateurs réalisés jusqu'à ce jour: les ascenseurs verticaux et les plans inclinés. Avec la pente d'eau, la masse d'eau sur laquelle flotte le bateau n'est pas transportée dans un récipient, mais cette masse d'eau est déplacée dans un tronçon de canal en pente qui assure la continuité entre le bief amont et le bief aval.

On voit sur la figure 1 :

- la bouchure mobile appelée "masque" qui déplace le long du canal en pente appelé "rigole" la masse d'eau en forme de coin sur lequel flotte le bateau;

- l'engin moteur qui pousse le masque, l'ensemble masque + engin moteur constituant ce que l'on appelle le "bouclier moteur".

Le cycle des opérations est le suivant :

- un bateau montant se présente à la tête aval ;

- on lève le masque pour le laisser entrer dans le coin d'eau;

- on descend ensuite le masque ;

- le bouclier moteur démarre et remonte le coin d'eau jusqu'à la tête amont ;

- lorsque le niveau du coin d'eau atteint celui du bief amont, la vanne qui délimite ce bief s'efface permettant la sortie du bateau montant et ensuite l'entrée du bateau avalant.

A noter que lorsqu'il s'agit d'un convoi et non d'un automoteur, ce qui est le plus fréquent en gabarit international, il est inutile de fractionner ce convoi dans le cas d'une pente d'eau, contrairement aux elévateurs et aux écluses.
L'histoire de la pente d'eau remonte à près de $15 \mathrm{ans}$ et il est inutile de rappeler le rôle déterminant qu'a joué dans sa mise au point le Professeur Aubert qui m'a chargé de l'excuser de n'avoir pu assister à cette session de la Société Hydrotechnique de France.

Cette histoire a commencé par une première phase d'études portant essentiellement sur le comportement dynamique du coin d'eau :

- d'abord études diverses dont l'étude mathématique du phénomène réalisée par le laboratoire de Chatou; - construction à Vénissieux et expérimentation d'un modèle réduit au $1 / 10$ par rapport à une installation sur canal à gabarit intemational (soit pour une largeur de canal de $12 \mathrm{~m}$ );

Cette première phase de développement a abouti à la conclusion que le comportement dynamique du coin d'eau pouvait être très aisément maîtrisé.

La décision a alors été prise d'appliquer pour la première fois le procédé sur des canaux en exploitation de type Freycinet, c'est-à-dire pour des bateaux de 350 tonnes.

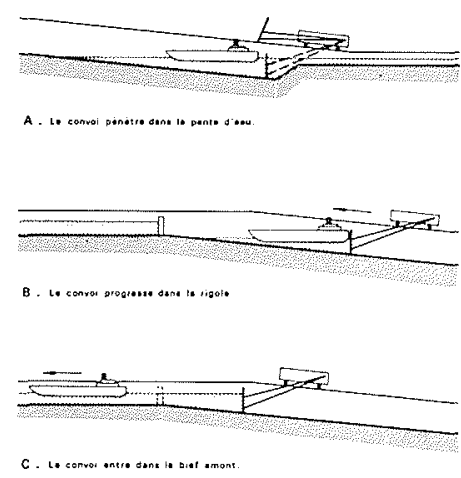

Figure 1. - Fonctionnement d'une pente d'eau. 


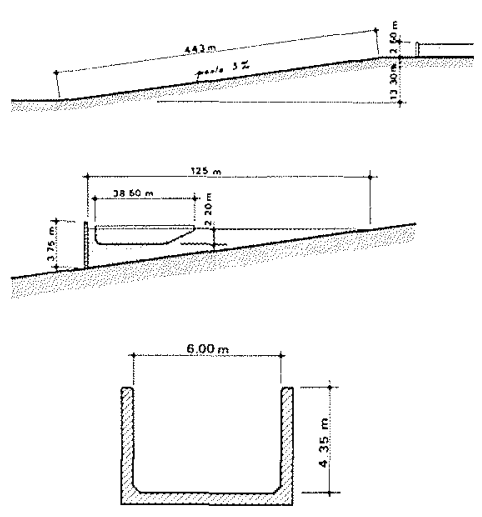

Figure 2. - Caractéristiques de Montech.

La modernisation du canal latéral à la Garonne ayant été entreprise, la Direction des Ports Maritimes et Voies Navigables décida de réaliser une pente d'eau sur le site de Montech pour le remplacement des 5 écluses existantes. La réalisation de l'ouvrage fut confiée par le Service de la Navigation de Toulouse au groupement S.G.T.E.-SPIE Batignolles (S.G.T.E. agissant comme bureau d'études ensemblier et SPIE Batignolles comme entrepreneur de Génie Civil).

Les caractéristiques principales de l'ouvrage de Montech (fig. 2) sont les suivantes :

\section{Caractéristiques de la rigole}

$\begin{array}{lc}\text { Hauteur de la chute } & 13,30 \mathrm{~m} \\ \text { Longueur de la rigole } & 443 \mathrm{~m} \\ \text { Pente } & 3 \% \\ \text { Largeur } & 6 \mathrm{~m}\end{array}$

\section{Dimensions du coin d'eau}

Hauteur devant le masque Longueur

$$
3,75 \mathrm{~m}
$$$$
125 \mathrm{~m}
$$

$\mathrm{Ce}$ coin d'eau permet le déplacement d'un automoteur de 350 tonnes $(38,50 \mathrm{~m} \times 5,50 \mathrm{~m} \times 2,20 \mathrm{~m})$.

Le bouclier moteur est constitué par :

- une automotrice à propulsion diesel de $1000 \mathrm{CV}$ roulant sur chaque berge sur roues et pneumatiqués; - une structure de liaison rigide de ces deux automotrices ;

- le masque et son pare-chocs supporté par cette structure.

Cet ouvrage inauguré en 1973 fonctionne depuis à la satisfaction de ses utilisateurs.

Par la suite, dans le cadre des études de la liaison Seine-Nord, le Service Central Technique des Ports Maritimes et Voies Navigables a confié à la S.G.T.E., fin 1977, l'étude d'un bouclier moteur pour pentes d'eau au gabarit international.

Cette étude qui fait l'objet du présent exposé a été réalisée en étroite collaboration entre le Service Central Technique et la S.G.T.E.

Elle a comporté deux phases.

$\mathrm{Au}$ cours de la première phase, furent examinées les différentes solutions envisageables pour le système de propulsion.

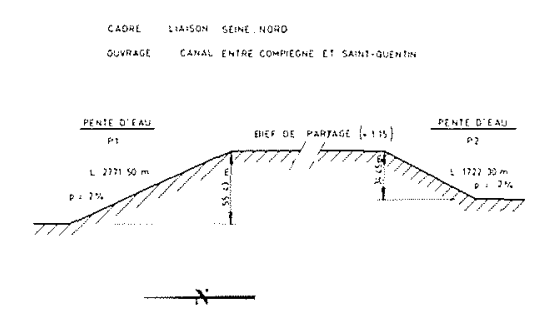

Figure 3. - Données géographiques de l'étude.

Au cours de la deuxième phase, il a été établi l'avant projet détaillé du système propulsif retenu.

Le projet Seine-Nord nécessitera la construction d'un canal de $135 \mathrm{~km}$ entre Compiègne et Saint-Quentin. Le bief de partage à la cote +115 (fig. 3 ) serait encadré par deux pentes d'eau :

- au Sud, la rigole aura une longueur de 1.722 mètres en pente $2 \%$, soit une dénivellation de 34,45 mètres. - au Nord, la rigole aura une longueur de 2.771 mètres en pente $2 \%$, soit une dénivellation de 55,43 mètres. Les deux boucliers seront évidemment identiques.

\begin{tabular}{|c|c|c|c|c|}
\hline CONvOIS & $\begin{array}{l}\text { Dit de } 5000 \text { Tonnes } \\
\text { (en tait } 5600 \text { Torness) }\end{array}$ & $\angle 000$ Tonnes & 800 Tonnes & 380 Tonnes \\
\hline ENFONCEMENT & $350 \mathrm{~m}$ & $300 \mathrm{~m}$ & $250 \mathrm{~m}$ & $220 \mathrm{~m}$ \\
\hline COMPOSIION & $\begin{array}{c}\text { 1 POUSSEUR } \\
2 \text { BARGES G5 GABBARII }\end{array}$ & $\begin{array}{c}1 \text { POUSSEUR } \\
2 \text { EARGE5 } \sigma^{\circ} \text { GABARII }\end{array}$ & $\begin{array}{l}2 \text { AUTOMOTEURS } \\
\text { OE } 3850 \mathrm{~m}\end{array}$ & $\begin{array}{l}1 \text { AUTOMOTEUR } \\
\text { DE } 38.50 \mathrm{~m}\end{array}$ \\
\hline LONGUEUR & $175 \mathrm{~m}$ & $175 \mathrm{~m}$ & $77 \mathrm{~m}$ & $3850 \mathrm{~m}$ \\
\hline LARGEUR & $11<0 \mathrm{~m}$ & $11<0 m$ & & \\
\hline
\end{tabular}

$$
\begin{aligned}
& \text { IRAFIC INITIAL } 10 \mathrm{M} \text { DE TONNES SOIT } 3070 \text { CYCLES/ AN } \\
& \text { CROISSANCE . } 2 \% \text { L'AN } 501 \text { 19 } 19 \text { dE TONNES AU BOUT DE } 30 \text { ANS } \\
& \text { POUR } 5560 \text { CYCLES/AN } \\
& \text { SATURATION } 19 \text { CYCLES/JOUR SOIT } 5840 \text { CYCLES/AN OU } 22 \text { M DE YONNES }
\end{aligned}
$$

Figure 4. - Convois types et hypothèses de trafic.

Quatre types de convois (fig. 4) sont à considérer :

- le convoi dit de 5000 tonnes constitué d'un pousseur et de 2 barges:

tirant d'eau $3,50 \mathrm{~m}$,

longueur totale $175 \mathrm{~m}$

- le convoi de 4000 tonnes. Le même que le précédent sauf le tirant d'eau $(3 \mathrm{~m})$;

- le convoi de 800 tonnes constitué par 2 automoteurs de $38,50 \mathrm{~m}$,

tirant d'eau $2,50 \mathrm{~m}$;

- l'automoteur de $38,50 \mathrm{~m}$.

Le tra fic initial est fixé à $10 \mathrm{Mt}$.

Croissance $2 \%$ l'an jusqu'à saturation du canal à $22 \mathrm{Mt}$.

Sur le tableau suivant (fig. 5) figurent les caractéristiques des coins d'eau nécessaires pour le déplacement de chacun de ces convois. 


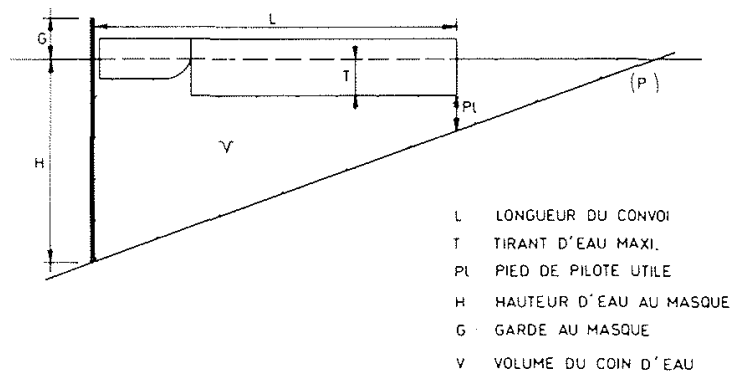

\begin{tabular}{|c|c|c|c|c|}
\hline & $\begin{array}{c}\text { CONVOI } \\
5000 \mathrm{~T}\end{array}$ & $\begin{array}{c}\text { CONVOI } \\
400 \mathrm{~T}\end{array}$ & $\begin{array}{c}\text { CONYOI } \\
800 \mathrm{~T}\end{array}$ & $\begin{array}{c}\text { BATEAU } \\
380 \mathrm{~T}\end{array}$ \\
\hline $\mathrm{L}$ & $175 \mathrm{~m}$ & $175 \mathrm{~m}$ & $72 \mathrm{~m}$ & $3850 \mathrm{~m}$ \\
\hline $\mathrm{T}$ & $350 \mathrm{~m}$ & $300 \mathrm{~m}$ & $250 \mathrm{~m}$ & $220 \mathrm{~m}$ \\
\hline$H$ & $730 \mathrm{~m}$ & $681 \mathrm{~m}$ & $611 \mathrm{~m}$ & $339 \mathrm{~m}$ \\
\hline $\mathrm{PI}$ & $0.30 \mathrm{~m}$ & $031 \mathrm{~m}$ & $0.37 \mathrm{~m}$ & $042 \mathrm{~m}$ \\
\hline$G$ & $0.40 \mathrm{~m}$ & $089 \mathrm{~m}$ & $329 \mathrm{~m}$ & $631 \mathrm{~m}$ \\
\hline$V$ & $15987 \mathrm{~m}^{3}$ & $13913 \mathrm{~m}^{3}$ & $5835 \mathrm{~m}^{3}$ & $346 \mathrm{~m}^{3}$ \\
\hline
\end{tabular}

Figure 5. - Coins d'eau caractéristiques.

Les volumes de ces coins d'eau s'échelonnent entre $3500 \mathrm{~m}^{3}$ et $16000 \mathrm{~m}^{3}$.

La hauteur d'eau au masque varie de 3,40 mètres à 7,30 mètres.

La dynamique de mouvement qu'il y a lieu de considerer est variable pour chaque convoi. Le tableau projeté (fig. 6) donne les valeurs des vitesses et des accélérations 1 la montée et à la descente, ainsi que l'accélération au redémarrage en côte après un éventuel arrêt au cours de la montée.

La puissance est déterminée par le déplacement du convoi de 4400 tonnes à la vitesse de $2 \mathrm{~m} / \mathrm{s}$. Pour les convois plus petits la vitesse peut être augmentée jusqu'a $3 \mathrm{~m} / \mathrm{s}$ (limite fixée par la tenue des pneumatiques).

Les lois de mouvement propres à chaque convoi ont té déterminées en fonction des caractéristiques dynamiques qui viennent d'être présentées.

Il en résulte d'une part des durées de fonctionnement 8 échelonnant entre 16 minutes et 28 minutes.

Et d'autre part les valeurs des différents efforts à développer. On distingue l'effort en marche continue, leffort maximum qui se produit, soit au redémarrage en cote, soit en fin de phase d'accélération, l'effort nominal de freinage pour la descente à vitesses constante, et leffort maximum de freinage pour l'arrêt du plus grand convoi avec une décélération de $5 \mathrm{~cm} / \mathrm{s}^{2}$. La puissance naximum nécessaire est de $7876 \mathrm{kw}$. Elle correspond au déplacement du convoi de 4400 tonnes à $2 \mathrm{~m} / \mathrm{s}$.

Le bouclier moteur (fig. 7) est de type portique. II repose sur deux files de 10 roues, de part et d'autre de la rigole. Chaque file de roues supporte une poutre de rive. Les deux poutres de rive sont solidarisées par deux traverses. La traverse arrière encaisse les efforts de poussée du masque. La traverse avant abrite le mécanisme de levage du masque.
1-VITESSES ET ACCELERATIONS

\begin{tabular}{|c|c|c|c|c|c|}
\cline { 2 - 6 } \multicolumn{1}{c|}{} & $\begin{array}{c}\text { CONVOI } \\
5000 \mathrm{t}\end{array}$ & $\begin{array}{c}\text { CONVOI } \\
4400 \mathrm{t}\end{array}$ & $\begin{array}{c}\text { CONVOI } \\
300 \mathrm{t}\end{array}$ & $\begin{array}{c}\text { BATEAU } \\
380 \mathrm{t}\end{array}$ & A VIDE \\
\hline$V_{M}$ & $1,76 \mathrm{~m} / \mathrm{s}$ & $2 \mathrm{~m} / \mathrm{s}$ & $3 \mathrm{~m} / \mathrm{s}$ & $3 \mathrm{~m} / \mathrm{s}$ & $3 \mathrm{~m} / \mathrm{s}$ \\
\hline$V_{0}$ & $2 \mathrm{~m} / \mathrm{s}$ & $2 \mathrm{~m} / \mathrm{s}$ & $3 \mathrm{~m} / \mathrm{s}$ & $3 \mathrm{~m} / \mathrm{s}$ & $3 \mathrm{~m} / \mathrm{s}$ \\
\hline$\gamma_{M}$ & $0,0155 \mathrm{~m} / \mathrm{s}^{2}$ & $0,02 \mathrm{~m} / \mathrm{s}^{2}$ & $0,045 \mathrm{~m} / \mathrm{s}^{2}$ & $0,025 \mathrm{~m} / \mathrm{s}^{2}$ & $0,00 \mathrm{~m} / \mathrm{s}^{2}$ \\
\hline$\gamma_{0}$ & $0,0155 \mathrm{~m} / \mathrm{s}^{2}$ & $0,02 \mathrm{~m} / \mathrm{s}^{2}$ & $0,065 \mathrm{~m} / \mathrm{s}^{2}$ & $0,045 \mathrm{~m} / \mathrm{s}^{2}$ & $0,00 \mathrm{~m} / \mathrm{s}^{2}$ \\
\hline$\gamma_{R}$ & $0,01 \mathrm{~m} / \mathrm{s}^{2}$ & $0,01 \mathrm{~m} / \mathrm{s}^{2}$ & $0,01 \mathrm{~m} / \mathrm{s}^{2}$ & $0,01 \mathrm{~m} / \mathrm{s}^{2}$ & $001 \mathrm{~m} / \mathrm{s}^{2}$ \\
\hline
\end{tabular}

2-DUREES DE FONCTIONNEMENT

\begin{tabular}{|l|c|c|c|c|c|}
\cline { 2 - 6 } \multicolumn{1}{c|}{} & $\begin{array}{c}\text { CONVO } \\
5000 \mathrm{t}\end{array}$ & $\begin{array}{c}\text { CONVOI } \\
4 \angle 00 \mathrm{t}\end{array}$ & $\begin{array}{c}\text { CONVOI } \\
800 \mathrm{t}\end{array}$ & $\begin{array}{c}\text { BATEAU } \\
380 \mathrm{t}\end{array}$ & A VIDE \\
\hline $\begin{array}{l}1 / 2 \mathrm{CYCLE} \\
\text { MONTEE }\end{array}$ & $28 \mathrm{mn} \mathrm{i5s}$ & $25 \mathrm{mn} 13 \mathrm{~s}$ & $17 \mathrm{mn} 03 \mathrm{~s}$ & $16 \mathrm{mn} 56 \mathrm{~s}$ & $16 \mathrm{mn} 33 \mathrm{~s}$ \\
\hline $\begin{array}{l}1 / 2 \text { CYCLE } \\
\text { DESCENTE }\end{array}$ & $28 \mathrm{mn}$ & $27 \mathrm{~mm} 55 \mathrm{~s}$ & $18 \mathrm{ma} 67 \mathrm{~s}$ & $18 \mathrm{mn} 11 \mathrm{~s}$ & $36 \mathrm{mn} 33 \mathrm{~s}$ \\
\hline
\end{tabular}

3-EFFORTS A DEVELOFPER

\begin{tabular}{|c|c|c|c|c|c|}
\hline $\begin{array}{l}\text { EFFORT } A \text { LA JANTE } \\
\text { TOTAL (en tonnes) }\end{array}$ & $\begin{array}{l}\text { CONvol } \\
5000 t\end{array}$ & $\begin{array}{l}\text { CONVOI } \\
4400 t\end{array}$ & $\begin{array}{l}\text { CONVOI } \\
800 t\end{array}$ & $\begin{array}{c}\text { BAIEAU } \\
380 \mathrm{t}\end{array}$ & A VIDE \\
\hline EFFORT EN MARCHE CORTINUE & $362 \mathrm{t}$ & 3211 & 1594 & $m$ & $37 \mathrm{k}$ \\
\hline $\begin{array}{l}\text { EFFORT HAXIMUM AU } \\
\text { REDEMARRAE EN COTE OU } \\
\text { EFORT ENAFE OE PHASE } \\
\text { D'ACCELERATIOH }\end{array}$ & 6281 & 3824 & $201:$ & $146 \mathrm{t}$ & $70 \mathrm{~s}$ \\
\hline $\begin{array}{l}\text { EFFORT NOMINAL OE FREINAGE } \\
\text { EN DESCEMTE } \mathrm{a} V=\mathrm{V}=\end{array}$ & $323 \mathrm{t}$ & $238 t$ & $127 t$ & $80 \mathrm{t}$ & 13: \\
\hline $\begin{array}{l}\text { EFFORT MOMIHAL EXCEPTONHEL } \\
\text { (FREINAGE) }\end{array}$ & 6531 & & & & \\
\hline
\end{tabular}

4. PUISSANCE INSTALLEE

$$
\begin{aligned}
& -P_{n}=7876 \mathrm{~kW}(10.700 \mathrm{ch}) \\
& \text { ELLE CORRESPONO AU DEPLACEMENT OU CONVOI DE } \angle L O O S \\
& \text { A } 2 \mathrm{~m} / \mathrm{s} \text { LORS OE LA MONTEE POUR UN RENDEMENT PROPULSIF } \\
& \text { glogat de o, }
\end{aligned}
$$

Figure 6. - Caractéristiques dynamiques.

De l'avant à l'arrière, on trouve donc la cabine de pilotage, la traverse avant, la salle des machines, la traverse arrière.

La chaîne de traction (fig. 8) est la suivante :

- l'énergie est captée sur le réseau par l'intermédiaire d'une sous station;

- elle est transmise à l'engin à travers un système caténaire pantographe ;

- cette énergie est transformée et traitée dans la salle des machines avant d'être distribuée aux moteurs qui actionnent les roues par l'intermédiaire d'un réducteur; - plusieurs dispositifs de freinage sont prévus.

Le fonctionnement de l'engin est donc caractérisé par 5 fonctions principales: la fonction alimentation, la fonction captation de courant, la fonction traction, la fonction freinage et les fonctions auxiliaires.

Nous allons examiner successivement ces 5 fonctions.

\section{- La fonction alimentation :}

Le poste de transformation alimente l'installation par deux arrivées aériennes en deux boucles de $60 \mathrm{kV}$. Il comporte :

- deux cellules "arrivées lignes" (normale et secours); 

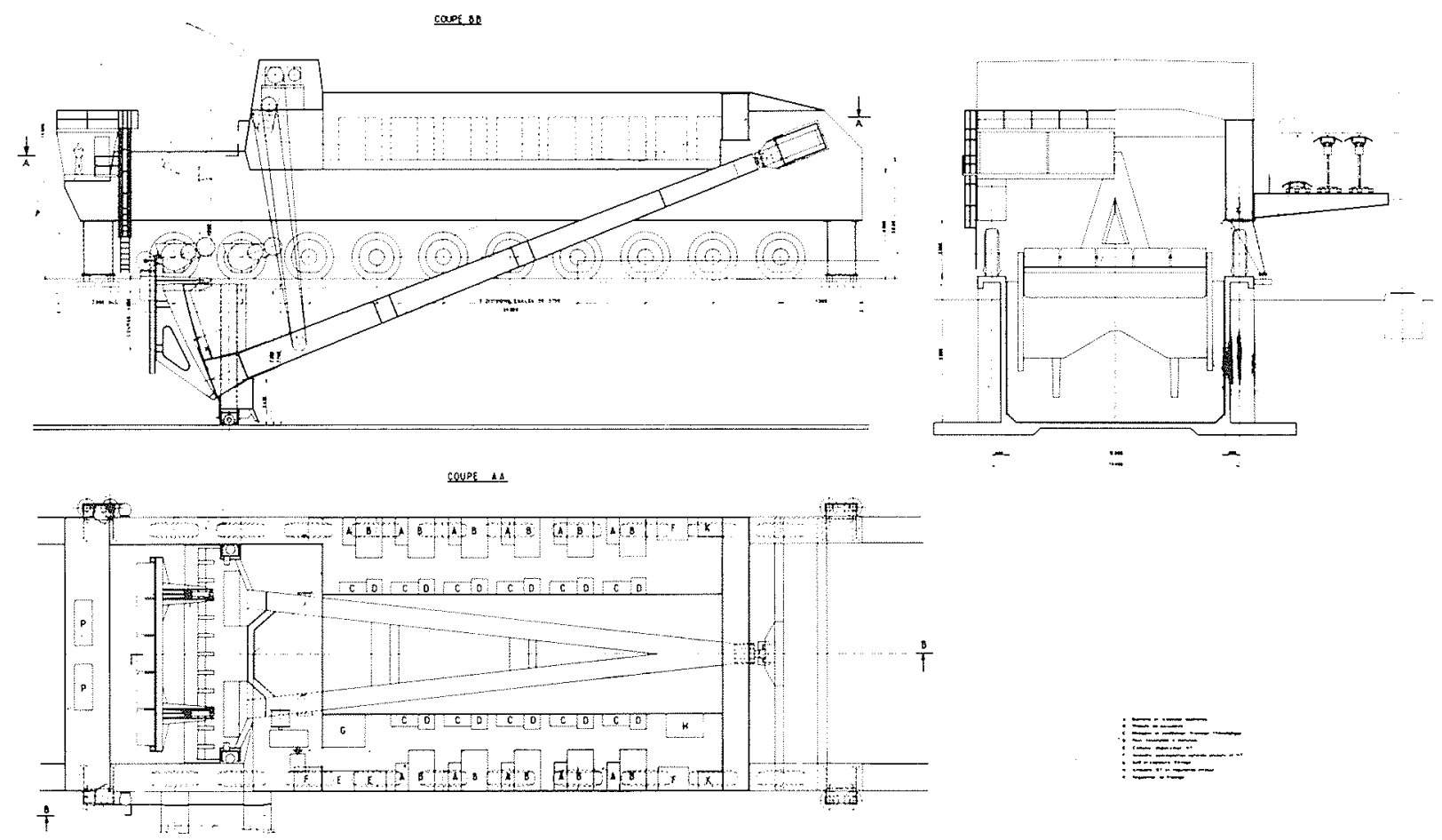

Figure 7

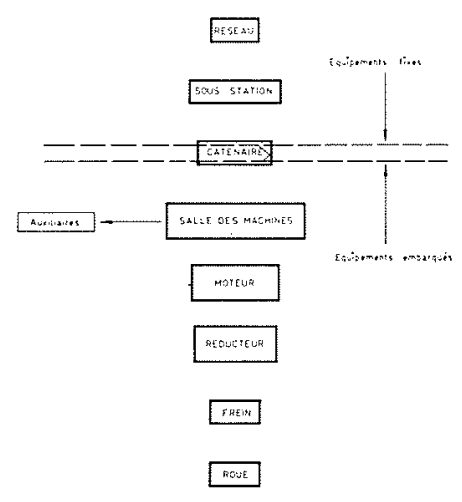

Figure 8. - Chaîne de traction.

- deux cellules "départs transformateurs" (normale et secours) ;

- un jeu de barres sectionnable (pour permettre l'entretien sans arrêt de fonctionnement);

- et deux transformateurs 60/20 kv 15 MVA (normal et secours).

Un bâtiment regroupe les tableaux $20 \mathrm{kV}$, les accessoires de commande et relayage, l'armoire redresseur et batterie, les accessoires et les groupes diesel pour les secours auxiliaires de sécurité.

\section{- La fonction captation de courant:}

L'alimentation de l'engin se fait par une ligne triphasée de $20 \mathrm{kV}$ le long du chemin de roulement suivant le principe du fil de contact simple, type S.N.C.F. Nous avons donc 3 fils de contact, un par phase. Trois pantographes spéciaux intégrés au pousseur assureront la prise de courant; deux jeux sont prévus (un normal et un secours). La ligne est à la hauteur réglementaire de $8 \mathrm{~m} 15$.

\section{- La fonction traction :}

La salle des machines abrite les 11 ensembles de transmission de la puissance électrique (10 en service et 1 en secours).

$\mathrm{Au}$ sein de chaque ensemble de transmission on trouve :

- les cellules de transformation du $20 \mathrm{kV}$;

- la commande (redresseur/onduleur) des moteurs;

- l'excitation avec redresseur (ou batterie de secours) et le hacheur.

L'engin repose sur 20 pieds sur lesquels sont montés les groupes moto-réducteurs, les freins mécaniques et les disques d'inertie. Les disques d'inertie sont prévus pour récupérer la différence de décelération entre le bouclier moteur et le coin d'eau en cas de coupure brusque d'alimentation à la montée.

Les 20 moteurs de traction développent chacun $370 \mathrm{kw}$ à $1200 \mathrm{t} / \mathrm{mn}$ pour une tension d'alimentation de 590 volts et une intensité de 695 ampères. Le réducteur est calculé pour transmettre le couple maximum sur 18 roues au lieu de $20 \ldots$ Son rapport de réduction est de 87.

Pour un poids total de l'engin de 900 tonnes, chaque pneu supporte 45 tonnes normalement et 50 tonnes dans le cas d'un fonctionnement sur 18 roues.

Les pneumatiques choisis ont un rayon libre de $1,35 \mathrm{~m}$. La vitesse limite adoptée pour la tenue des pneus $3 \mathrm{~m} / \mathrm{s}$ en fonctionnement usuel ramené à $2 \mathrm{~m} / \mathrm{s}$ sous 18 roues. 


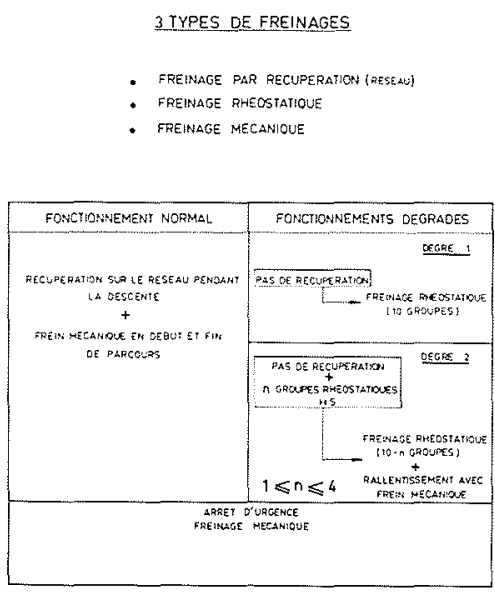

Figure 9. - Fonction freinage.

\section{- La fonction freinage :}

La fonction freinage est assurée par trois systèmes distincts (fig. 9):

- freinage par récupération ;

- freinage rhéostatique ;

- freinage mécanique.

En fonctionnement normal, au cours de la descente on utilise le freinage par récupération sur le réseau: les moteurs fonctionnent alors en génératrices et l'énergie cinétique du pousseur est transformée en énergie renvoyée sur le réseau.

En début et en fin de trajet on utilise un freinage mécanique à disques.

En fonctionnement dégradé, c'est-à-dire dans le cas d'impossibilité de renvoi de la tension sur le réseau, on utilise le freinage rhéostatique. Ce freinage rhéostatique est constitué par 10 résistances embarquées, capables chacune de dissiper l'énergie des deux moteurs de "l'essieu" qui lui est affecté.

La structure modulaire rend très improbable la panne simultanée de ces 10 groupes rhéostatiques; on peut donc envisager un fonctionnement dégradé de degré 2 , où un ou plusieurs groupes rhéostatiques sont en panne et où le freinage mécanique à disques vient compenser le manque correspondant pratiquement il est encore possible de descendre avec quatre groupes rhéostatiques hors service.

En arrêt d'urgence, on utilise le freinage mécanique. Les 20 disques sont dimensionnés pour freiner le plus grand convoi avec une décélération de $5 \mathrm{~cm} / \mathrm{s}^{2}$. L'engin s'arrête alors sur une longueur de $40 \mathrm{~m}$ (c'est-à-dire sur sa longueur).

- Les fonctions auxiliaires, c'est-à-dire :

- le guidage de l'engin sur la rigole ;

- la sustentation et le levage du masque;

- le système d'étanchéité masque-rigole;

- le pare-choc;

- la cabine de pilotage.

Le guidage de l'engin sur la rigole est nécessaire d'abord pour lutter :
- contre la poussée de carrossage des pneus porteurs et le vent transversal qui peuvent induire des efforts transversaux jusqu'à 13 tonnes ;

- il y a ensuite les dissymétries des efforts de traction et de freinage qui sont négligeables;

- la condition déterminante est finalement l'éventualité d'un blocage de roue qui conduit à un effort de 30 tonnes par côté ;

- des jauges extensiométriques déclencheront le freinage d'urgence pour un effort dépassant 20 tonnes par côté ; - le guidage envisagé est réalisé par 4 jambes de forces, deux de chaque côté de la rigole. Chacune porte deux pneus de type métro.

Sustentation et levage du masque: le masque doit pouvoir être relevé et abaissé systématiquement à la tête aval de l'installation. Son poids est de 100 tonnes ( 60 tonnes pour la vanne et 40 tonnes pour le bras). Un système par câble et treuil est prévu pour son levage.

Le dispositif d'étanchéité masque-rigole est constitué par des rouleaux cylindriques en néoprène portés par le masque et qui roulent sans glisser sur les faces internes des bajoyers et sur le radier. Ces rouleaux sont logés dans des boitards.

Les rouleaux latéraux sont appliqués sur la rigole par déplacement des boitards à l'aide de vérins.

Les rouleaux inférieurs sont appliqués sur le radier par leur propre poids.

Des injections d'eau dans les boitards empêchent toute intrusion de corps étrangers dans le système d'étanchéité. Pour le plus grand convoi, les fuites sont estimées à $1000 \mathrm{~m}^{3} / \mathrm{h}$.

Cette photo montre un rouleau latéral en position masque levé.

La poutre pare-chocs doit assurer l'appui, voire l'encaissement des légers chocs dûs au mouvement des convois amarrés, ainsi que le choc d'un convoi arrivant à $1 \mathrm{~m} / \mathrm{s}$. D'où un effort de 50 tonnes que les ressorts amortisseurs prévus peuvent encaisser.

Le pare-chocs doit en outre être adaptable aux dimensions des différents convois. Sa face avant dispose donc d'un appui coulissant sur une hauteur de 5,30 m.

\section{La cabine de pilotage rassemble:}

- le tableau de navigation avec les commandes du masque, du système d'amarrage, des étanchéités, du pare-chocs;

- le tableau de contrôle du pousseur avec les commandes et contrôles de son mouvement ;

- le tableau de commandes des auxiliaires.

On trouve également au sein de la cabine de pilotage : les systèmes de télécommande des portes amont et aval, la télémesure des niveaux de l'eau dans la rigole et dans les biefs et les communications radios et réseau de télévision.

Au niveau de la disponibilité, la subdivision en 10 groupes de traction indépendants +1 en secours permet d'assurer la maintenance courante sans perturber le fonctionnement de l'engin. Un système plaque-vérins de compression permet d'isoler une roue défaillante pour 
que l'engin continue son service de la joumee. Ce système, combiné à un chariot support sur rail, permet de procéder rapidement à un échange standard du groupe motoréducteur, qui pourra ainsi être réparé sans hâte dans un atelier de maintenance.

Les réparations importantes s'effectueront dans un atelier de maintenance qui assurera le magasinage des composants de rechange courants, le stockage des pièces d'usure et le magasinage des composants de roulage. Des aires de travail sont prévues pour le démontage des groupes motoréducteurs et pour le chargement des roues. Cet atelier fera fonction d'atelier principal et sera installé sur le site de la plus grande pente d'eau. Un atelier annexe plus réduit sera prévu sur le site de la deuxième.

A la suite de toute cette étude, le coût d'investissement pour un bouclier moteur de pente d'eau grand gabarit était estimé à 50 millions de francs en janvier 1978 (fig. 10)

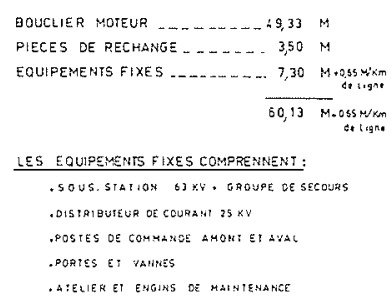

Figure 10. - Coûts d'investissement (base janvier 1978).

$\mathrm{Au}$ titre de l'investissement, il faut ajouter les pièces de rechange pour 3,5 millions et le coût des équipements fixes pour 7,3 millions, de sorte que le coût total d'investissements s'établissait à cette date à 60 millions de francs. Il faut y ajouter le coût de la ligne E.D.F. et le cout de réalisation du génie civil de la rigole soit au total 110 millions de francs et donc un coût de $20 \%$ inférieur à celui d'une écluse à bassin d'épargne.

\begin{tabular}{|c|c|c|c|c|c|}
\hline & \begin{tabular}{|r|}
2000 cycless \\
$/ \mathrm{an}$
\end{tabular} & $\begin{array}{r}3000 \text { cyclos } \\
/ 2 n \\
\end{array}$ & \begin{tabular}{|r|}
4000 cycles \\
/an \\
\end{tabular} & $\begin{array}{r}5000 \text { cycles } \\
/ 20 n\end{array}$ & $\begin{array}{r}5500 \mathrm{cycles} \\
/ \mathrm{m}\end{array}$ \\
\hline $\begin{array}{l}\text { COUT OES } \\
\text { CONSOMMATIONS } \\
\text { ENERGETIQUES(KF) }\end{array}$ & 2397 & 2710 & 3023 & 3325 & 3692 \\
\hline $\begin{array}{l}\text { COUT DES } \\
\text { PNEUMATIOUES } \\
\text { ET DES } \\
\text { ETANCHEITES(KF) }\end{array}$ & 299 & 448 & 597 & 747 & 822 \\
\hline $\begin{array}{l}\text { COUT DE } \\
\text { LA MAINENACE } \\
\text { COURANTEIKF) }\end{array}$ & 976 & 1122 & 1268 & 1614 & 1488 \\
\hline $\begin{array}{l}\text { COUT DU PERSONNE } \\
(K F)\end{array}$ & 1700 & 1700 & 2300 & 2300 & 2300 \\
\hline TOTAL & 5372 & 5980 & 7188 & 7796 & 8102 \\
\hline
\end{tabular}

Le tableau ci-dessus donne les coûts de fonctionnement. Nous avons distingué les coûts des consommations énergétiques (après consommation due à la récupération à la descente), des principales pièces d'usure, de la maintenance et des frais de personnel (base janvier 1978)

Il est à noter que la pente d'eau est la solution à consommation énergétique minimum. Par cycle:
$1800 \mathrm{kwh}$ pour la pente d'eau et plus de $3000 \mathrm{kwh}$ pour une écluse à bassin d'épargne.

En conclusion, l'étude entreprise courant 1978 par la S.G.T.E. pour le compte du Service Central Technique a ainsi permis de concevoir un bouclier moteur pour pente d'eau gabarit $12 \mathrm{~m}$ à propulsion électrique. Les dispositifs et matériels qui le constituent ont été choisis systématiquement dans des types de fabrication courants assurant un coût d'entretien minimum pour une fiabilité maximum.

D'autres systèmes de propulsion sont aussi possibles, notamment la propulsion par moteur hydraulique ce qui démontre la grande flexibilité du procédé.

Qu'il me soit permis de terminer cet exposé indigeste et austère par des considérations générales à partir de cet exemple de la pente d'eau sur de telles actions de recherches et développement.

Un autre exemple dans ce domaine de la Navigation Intérieure est celui de la mise au point du barrage-clapets à vérins immergé dont le premier exemplaire vient d'être réalisé sur la Seine à Denouval avec comme promoteur là encore le Professeur Aubert et auquel la S.G.T.E. s'est trouvée aussi associée.

Il est superflu de rappeler la nécessité vitale pour la France d'exporter et les difficultés actuelles de notre balance commerciale.

Les Pouvoirs Publics français bien qu'avec retard par rapport à ceux de nos concurrents étrangers ont pris conscience de l'importance stratégique de la profession de l'Ingénièrie à laquelle appartient la S.G.T.E. dans cette bataille de l'exportation, du fait notamment de son effet d'entraînement sur les autres professions. Or ce rôle stratégique, la profession de l'Ingéniérie le jouera d'autant plus efficacement et durablement qu'elle pourra disposer toujours de techniques que les autres, clients et concurrents étrangers, n'ont pas encore.

Là encore tout le monde a conscience que l'avance technologique est une question de survie pour des pays développés comme la France.

Ceci suppose que les Pouvoirs Publics, au moins dans le secteur de l'Infrastructure où l'Etat est le client unique, aillent au-delà d'une simple prise de conscience en mettant en cuvre une véritable politique de soutien de l'innovation au profit notamment de l'Ingénierie.

Cette politique doit consister d'abord à participer financièrement aux actions de recherche et développement pour la mise au point de techniques et procédés susceptibles d'être exportés. Cela a été le cas pour la pente d'eau par une collaboration tout à fait exemplaire avec le Ministère de l'Environnement et du Cadre de Vie et les différents Services de la Navigation.

Mais cette politique doit consister aussi à engager en priorité la première réalisation en France de ces procédés et techniques exportables, car le client étranger hésitera toujours à s'engager lui-même dans la réalisation d'un procédé si la Société d'Ingénierie qui le lui propose ne peut faire état d'une réalisation de ce procédé à laquelle elle aurait participé dans son propre pays.

Je terminerai donc en formulant le vœu que cette condition puisse être satisfaite dans le cas de ce procédé nouveau par la réalisation prochaine en France d'une pente d'eau de gabarit international. 